\title{
Malaria parasite clearance from patients following artemisinin-based combination therapy in Côte d'Ivoire
}

This article was published in the following Dove Press journal: Infection and Drug Resistance

\author{
Offianan Andre Toure' \\ Tiacoh N'Guessan Landry' \\ Serge Brice Assi ${ }^{2,3}$ \\ Antoinette Amany Kone' \\ Eric Adji Gbessi' \\ Berenger Aristide Ako' \\ Baba Coulibaly' \\ Bouakary Kone ${ }^{4}$ \\ Oumar Ouattara ${ }^{4}$ \\ Sylvain Beourou' \\ Alphonsine Koffi ${ }^{2}$ \\ Franck Remoue ${ }^{2,5}$ \\ Christophe Rogier 6
}

'Malariology Unit, Pasteur Institute of Côte d'Ivoire, Abidjan, Côte d'Ivoire; ${ }^{2}$ Malaria and Anopheles Research and Management Unit, Pierre Richet Institute, Bouake, Côte d'Ivoire; ${ }^{3}$ National Malaria Control Program, Bouake, Côte d'Ivoire; ${ }^{4}$ Department of Medicine, Health Care Center of Dar-Es-Salam, Bouake, Côte d'Ivoire; ${ }^{5}$ UMR 224-MIVEGEC, Research Development Institute, Montpellier, France; ${ }^{6}$ Army Health Department, Paris, France

Correspondence: Offianan Andre Toure Malariology Unit, Pasteur Institute of Côte d'lvoire, PO Box 490, Abidjan 0I, Côte d'lvoire

Email andre_offianan@yahoo.fr
Introduction: Parasite clearance is useful to detect artemisinin resistance. The aim of this study was to investigate parasite clearance in patients treated with artesunate + amodiaquine (AS + $\mathrm{AQ}$ ) and artemether + lumefantrine (AL): the two artemisinin-based combination therapies (ACTs) recommended in the first-line treatment of uncomplicated malaria in Côte d'Ivoire.

Methods: This study was conducted in Bouaké, Côte d'Ivoire, from April to June 2016. Patients aged at least 6 months with uncomplicated malaria and treated with AS + AQ or AL were hospitalized for 3 days, and follow-up assessments were performed on days 3, 7, 14, 21, 28, 35, and 42. Blood smears were collected at the time of screening, pre-dose, and 6-hour intervals following the first dose of administration until two consecutive negative smears were recorded, thereafter at day 3 and follow-up visits. Parasite clearance was determined using the Worldwide Antimalarial Resistance Network's parasite clearance estimator. The primary end points were parasite clearance rate and time.

Results: A total of 120 patients (57 in the AS + AQ group and 63 in the AL group) were randomized among 298 patients screened. The median parasite clearance time was 30 hours (IQR, 24-36 hours), for each ACT. The median parasite clearance rate had a slope half-life of 2.36 hours (IQR, 1.85-2.88 hours) and 2.23 hours (IQR, 1.74-2.63 hours) for AS + AQ and $\mathrm{AL}$, respectively. The polymerase chain reaction-corrected adequate clinical and parasitological response was $100 \%$ and $98.07 \%$ at day 42 for AS + AQ and AL, respectively.

Conclusion: Patients treated with AS + AQ and AL had cleared parasites rapidly. ACTs are still efficacious in Bouaké, Côte d'Ivoire, but continued efficacy monitoring of ACTs is needed. Keywords: Plasmodium falciparum, ACTs, parasite clearance, Côte d'Ivoire

\section{Introduction}

Malaria remains a serious public health problem in sub-Saharan Africa despite the reduction in morbidity and mortality observed in recent years. ${ }^{1}$ The advances in the control of malaria are partly related to the use of artemisinin-based combination therapies (ACTs) in almost all endemic countries in combination with other strategies.

However, the emergence and spread of artemisinin-resistant malaria parasite populations in Southeast Asian countries and the possibility of extension to other endemic areas are serious threats. ${ }^{2-4}$ The possibility that artemisinin resistance might spread or emerge independently elsewhere necessitates careful surveillance. ${ }^{5}$

The WHO recommends that the efficacy of the first- and second-line antimalarial drugs should be regularly assessed for early detection and prevention of the spread of resistant parasite populations. ${ }^{6}$ 
Several reliable methods exist currently to search for possible emergence of resistance to artemisinin derivatives. Surveillance of artemisinin resistance to date has relied on in vivo studies to measure early clearance of peripheral parasitaemia by microscopy and $\mathrm{k} 13$ propeller gene mutations. ${ }^{7}$

The phenotype of artemisinin resistance is characterized by a significant delay in parasite clearance following the initiation of therapy. ${ }^{8-10}$ Thus, many studies on the k13 propeller marker gene for resistance to artemisinin conducted in sub-Saharan Africa have shown no evidence of resistance mutation which is associated with artemisinin resistance in Southeast Asia. ${ }^{11-14}$

Therefore, parasite clearance rates are important measures of antimalarial drug efficacy and particularly important in the assessment of artemisinin resistance. The parasite clearance estimator (PCE) developed by Worldwide Antimalarial Resistance Network (WWARN) seemed to be an accurate and reliable method for the early detection of artemisinin resistance. ${ }^{15-17}$ This method is uses by sampling multiple times $(6,8$, or 12 hours) a day at measured time points to estimate the rate of parasite clearance.

A number of clinical trials have already been conducted in different parts of Côte d'Ivoire on artesunate + amodiaquine $(\mathrm{AS}+\mathrm{AQ})$ and artemether + lumefantrine (AL) efficacy and safety from 2005 to 2016 in Malaria Control Program sentinel sites. ${ }^{18-20}$ Results from these studies have provided evidence that the two ACTs remain efficacious in the treatment of Plasmodium falciparum uncomplicated malaria. Polymerase chain reaction (PCR)-corrected 28-day adequate clinical and parasitological response (ACPR) for each ACT was more than $95 \%$ with and no early clinical failures. However, in Bouake, which is one of the newest sentinel sites of the National Malaria Control Program (NMCP) of Côte d'Ivoire having high malaria transmission, no efficacy data of AS + AQ and AL are available since the use of these ACTs in this region. Therefore, this study was designed to assess parasite clearance and antimalarial drug efficacy in patients treated with AS + AQ and AL in Bouake, Center of Côte d'Ivoire.

\section{Patients and methods Study design and site}

The study was performed during a clinical trial conducted from April to June 2016 in Bouake to assess parasite clearance rate and time using WWARN's PCE. ${ }^{21}$ The study site is located in the center of Côte d'Ivoire, where malaria transmission is intense with recrudescence during the rainy season. The main vectors for malaria transmission in this area are Anopheles gambiae and Anopheles funestus. P. falciparum is the predominant malaria parasite, accounting for more than
$95 \%$ of malaria infections. ${ }^{18}$ Bouake region was the epicenter of the country's military crisis in 2002. During this period, the NMCP's guidelines for malaria treatment were not followed by physicians. Bouake is a cosmopolitan city with intense migratory movements of the populations. According to the latest population census in 2014, Bouake's population has reached more than half a million. The city of Bouake is the nation's second largest community and commercial and transportation hub of Cote d'Ivoire.

\section{Study population}

All patients aged at least 6 months with uncomplicated falciparum malaria were screened. The WHO's standard efficacy testing protocol was used. ${ }^{6}$ Briefly, patients with monospecific P. falciparum infestation confirmed by microscopy with parasite density between 2,000 and 200,000 asexual parasites/ $\mu \mathrm{L}$ of blood, axillary temperature of $\geq 37.5^{\circ} \mathrm{C}$, or history of fever over the past 24 hours, body weight $\geq 5 \mathrm{~kg}$, and who were able to take oral medications and follow study procedures, were included after informed consent was obtained from participant, parents, or legal guardian. Patients with severe malaria symptoms, symptoms of severe malnutrition, or chronic diseases or with mixed infection were excluded.

\section{Sample size}

The sample size was calculated using the WHO guidelines on the assessment of antimalarial drugs. ${ }^{6}$ Population size was determined according to the following criteria: the proportion of probable clinical failures with the antimalarial combinations studied should not be higher than $10 \%$, for a level of confidence of $95 \%$ and a precision of $10 \%$, taking into account patients who were excluded or lost to follow up. Using these criteria, a minimum of 50 patients was required in each treatment arm.

\section{Clinical procedures}

All included patients were hospitalized for 3 days (day 0 , 1,2 ), and follow-up assessments were performed on day 3 , $7,14,21,28,35$, and 42. Physical examination, vital signs, body temperature, and clinical assessment were performed at screening and at all follow-up visits. Body temperature was recorded at 6-hour intervals following the first dose of study medication until temperature normalized and remained normal for 24 hours and at every visit thereafter.

\section{Drug administration}

Patients were randomly assigned to receive either AS + AQ or AL. Both treatments were 3-day oral regimens dosed by 
weight according to the manufacturer's instructions: AS + AQ 5 to <9 kg: one tablet/day of AS $25 \mathrm{mg} / \mathrm{AQ} 67.5 \mathrm{mg}$; 9 to $<18 \mathrm{~kg}$ : one tablet/day of AS $50 \mathrm{mg} / \mathrm{AQ} 135 \mathrm{mg} ; 18$ to $<36 \mathrm{~kg}$ : one tablet/day of AS $100 \mathrm{mg} / \mathrm{AQ} 270 \mathrm{mg}$; $\geq 36 \mathrm{~kg}$ : two tablets/day of AS $100 \mathrm{mg} / \mathrm{AQ} 270 \mathrm{mg}$. AS + AQ were administered once daily in children and twice daily in adult participants.

AL tablet strength was $20 \mathrm{mg}$ artemether/120 mg lumefantrine: 5 to $<15 \mathrm{~kg}$ : one tablet/dose; 15 to $<25 \mathrm{~kg}$ : two tablets/dose; 25 to $<35 \mathrm{~kg}$ : three tablets/dose; $\geq 35 \mathrm{~kg}$ four tablets/dose. AL was administrated twice a day. If vomiting occurred within 30 minutes following the first drug dose, a redosing was performed. If the patient vomited within 30 minutes of repeated dosing, he or she was withdrawn from the trial and given rescue medication according to the NMCP's malaria treatment guidelines.

\section{Laboratory procedures}

\section{Parasitological assessments}

Thick/thin blood smears were collected at the time of screening, pre-dose, and at day 0- and 6-hour intervals following the first dose of administration until two consecutive negative smears were recorded, thereafter at day 3 and follow-up visits. Blood smears were prepared using Giemsa staining $(10 \%)$. Thick film was examined with a binocular microscope with an oil immersion objective lens to quantify the parasitaemia. Parasitaemia was measured by counting the number of asexual parasites and leucocytes in 200 high power fields based on a putative count of 8,000 leucocytes $/ \mathrm{mL}$ of blood.

Two qualified independent microscopists read all Giemsastained slides. Discordant readings were reexamined by a third qualified independent microscopist.

Discordance was defined as differences between the first and second microscopist regarding parasite density $>25 \%$, species diagnosis or any difference that affected recruitment or study outcome. Final parasite density was the average of the two most concordant counts.

\section{PCE}

The PCE established by WWARN was used to determine parasite clearance rate, parasite clearance time (PCT), slope half-life, and the time needed for parasitemia to fall by $50 \%, 90 \%, 95 \%$, and 99\% (PC50, PC90, PC95, and PC99, respectively).

WWARN's PCE is an uniform, reliable, and accurate method to estimate malaria parasite clearance based on the linear portion of the slope of the log-parasitaemia vs time relationship. ${ }^{15,21}$
This standardized approach provides information that enables the detection of early changes in $P$. falciparum sensitivity to artemisinins and support timely responses in treatment guidelines when needed.

\section{Parasite genotyping}

To distinguish between recrudescence and reinfection, blood spots on Whatman ${ }^{\circledR} 3 \mathrm{MM}$ filter paper (Whatman International Ltd, Maidstone, UK) were prepared before treatment and after day 7 in the event of reappearance of parasites, confirmed by microscopy. PCR genotyping was performed following the standard protocol as previously described on the basis of $P$. falciparum merozoite surface protein 1 ( $m s p l$ ), merozoite surface protein 2 ( $m s p 2$ ) and glutamate-rich protein (glurp). ${ }^{6,22,23}$ Possible outcomes were new infection or recrudescence. A "new infection" is a subsequent occurring parasitaemia in which all the alleles of the parasites from the posttreatment sample are different from those in the admission sample, for one or more loci tested. In a "recrudescence," at least one allele at each locus should be in common for both paired samples.

\section{Study end points}

The primary end points were PCT and parasite clearance rate. The PCT was defined as the time in hours from the first treatment dose to the first of two consecutive thick blood films that were negative for asexual $P$. falciparum parasites after checking 200 oil immersion fields. The secondary outcomes were the PCR-corrected ACPR at day 42, fever clearance times, time in hours from the start of treatment at which the temperature first decreased to $<37.5^{\circ} \mathrm{C}$ and remained below $37.5^{\circ} \mathrm{C}$ for 24 hours.

\section{Statistical analyses}

SPSS Version 17 (SPSS Inc., Chicago, IL, USA) was used for data management and analysis. Frequencies were compared by either chi-squared or Fisher's exact tests, as appropriate, and continuous variables by Student's $t$-tests.

Distribution of parasite clearance rate constants and slope half-lives were generated by the WWARN's PCE (http://www. wwarn.org/research/parasite-clearance-estimator).

\section{Ethical issues}

The study was conducted according to the local laws and regulations, International Conference on HarmonizationGood Clinical Practice (ICH-GCP). The protocol was reviewed and approved by the Comité National d'Ethique de la Recherche de Côte d'Ivoire [National Research Ethic Committee of Côte d'Ivoire]. Written informed consent was 
obtained from each participant or legal guardians before any sample collection. In the case of an illiterate patient, his/her thumb impression and signature of an independent witness were obtained. Patients providing assent were $<18$ year old who were able to understand and complied with study procedures in addition to parents' or legal guardians' written informed consent.

\section{Results}

\section{Trial profile}

The trial profile is shown in Figure 1. From April to June 2016, 298 patients with suspected malaria were screened for eligibility in the clinical trial. A total of 120 patients were eligible and randomized in the AS + AQ (57) and AL (63) groups. The reasons for ineligibility were low-density parasitaemia $(<2,000$ parasites $/ \mu \mathrm{L}$ of blood), very highdensity parasitaemia ( $>200,000$ parasites $\mu \mathrm{L}$ of blood), low probability of completing follow-up, inability to take oral drugs, and unwillingness to consent to the study. Six patients (two in the AS + AQ group and four in the AL group) defaulted as a result of withdrawal and/or lost to follow up. Primary end points were reached by 54 and 55 patients in the AS + AQ and AL groups, respectively.

\section{Baseline characteristics of study population}

The study population consisted of $44.4 \%$ males and $55.6 \%$ females in the AL group. In the AS + AQ group, $40.3 \%$ of patients were males. The median age was $7 \pm 0.67$ years in the AL group and $8.7 \pm 1.0$ years in the AS +AQ group. Mean weight was $22.1 \pm 1.65 \mathrm{~kg}$ in the AL group and $23.3 \pm 1.5 \mathrm{~kg}$ in the AS + AQ group. Both treatment groups were comparable in terms of baseline demographic, clinical, and laboratory characteristics except for hemoglobin and age (Table 1).

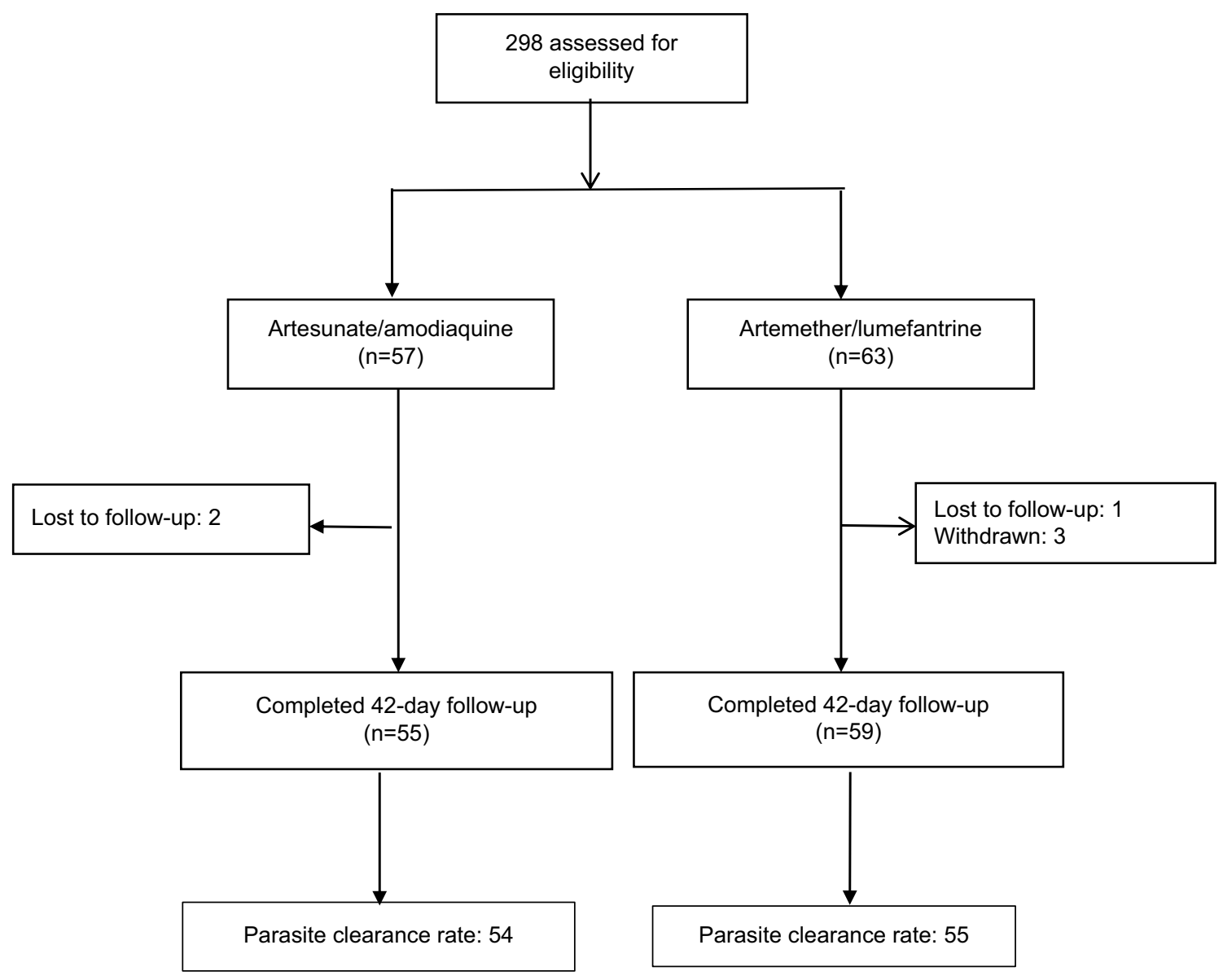

Figure I Trial profile. 


\section{Primary end points}

The parasite clearance rate was assessed in 54 and 55 patients in the AS + AQ group and AL group, respectively. The median PCT in this study was 30 hours (IQR, 24-36 hours), for each ACT, and the median parasite clearance rate had a slope half-life of 2.36 hours (IQR, 1.85-2.88 hours) and 2.23 hours (IQR, 1.74-2.63 hours) for AS + AQ and AL, respectively (Table 2).

\section{Secondary end points}

No early treatment failure (ETF) was observed in either of the study groups. Day 42 crude ACPRs were $96.4 \%$ and $83.3 \%$ in the AS + AQ and AL groups, respectively. Twelve patients who completed 42-day follow-up had reappearance of parasitaemia (ten in the AL group, two in the AS + AQ group) between days 14 and 42 after the initial clearance of parasitaemia. PCR genotyping was successfully performed on all blood samples. PCR-corrected ACPR at day 42 after PCR correction in per-protocol population was $100 \%$ and 98.07\% for AS + AQ and AL, respectively. This rate was $100 \%$ for each ACTs at day 28. Among those febrile on inclusion, the median fever clearance time was 6 hours (IQR, 6-6 hours) for each drug (Table 2).

\section{Discussion}

ACT as recommended by the WHO for the treatment of uncomplicated P. falciparum malaria has been adopted as the first-line therapy in most malaria-endemic countries.

Table I Baseline demographic characteristics

\begin{tabular}{|c|c|c|c|}
\hline Parameters & AL $(n=63)$ & $A S+A Q(n=57)$ & P-value \\
\hline Sex, n (\%) male & $28(44.4)$ & $23(40.3)$ & 0.78 \\
\hline Age (years), mean \pm SD & $7.0 \pm 0.67$ & $8.7 \pm 1.0$ & $0.16^{\mathrm{a}}$ \\
\hline \multicolumn{4}{|l|}{ Age group } \\
\hline $\mathrm{I}-5$ years, $\mathrm{n}(\%)$ & $30(47.6)$ & $17(29.8)$ & 0.07 \\
\hline 6-15 years, n (\%) & $27(42.8)$ & $38(66.6)$ & 0.01 \\
\hline$>15$ years, $n(\%)$ & $06(09.5)$ & $02(03.5)$ & 0.34 \\
\hline Weight $(\mathrm{kg})$, mean $\pm \mathrm{SD}$ & $22.1 \pm 1.65$ & $23.3 \pm 1.5$ & $0.59^{b}$ \\
\hline Pf. (parasitemia/mL), geometric mean \pm SD & $71,320 \pm 2.8$ & $59,437 \pm 2.8$ & $0,16^{b}$ \\
\hline Anemia (hemoglobin level <10), n (\%) & $20(31.7)$ & $10(17.5)$ & $0.1 I^{b}$ \\
\hline Fever (axillary temperature $\geq 37.5^{\circ} \mathrm{C}$ ), $\mathrm{n}(\%)$ & $63(100)$ & $57(100)$ & $\mathrm{I}^{\mathrm{b}}$ \\
\hline Body temperature among febrile patients $\left({ }^{\circ} \mathrm{C}\right)$, mean \pm SD & $39.2 \pm 0.08$ & $39.1 \pm 0.07$ & $0.30^{\mathrm{b}}$ \\
\hline
\end{tabular}

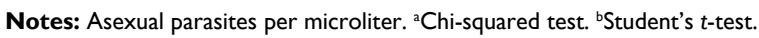

Abbreviations: AL, artemether + lumefantrine; AS + AQ, artesunate + amodiaquine; Pf., Plasmodium falciparum.

Table 2 Primary and secondary end points

\begin{tabular}{|c|c|c|c|}
\hline End point & $A L(n=63)$ & $A S+A Q(n=57)$ & $P$-value \\
\hline \multicolumn{4}{|l|}{ Primary end point ${ }^{\mathrm{a}}$} \\
\hline Parasite clearance rate $(n)^{b}$ & 55 & 54 & \\
\hline Lag phase (hours), (n); median (range) & $(29) ; 6.0(6-12)$ & $(31) ; 6.0(6-12)$ & 0.77 \\
\hline Parasite clearance rate constant(per hour), median (IQR) & $0.29(0.24-0.37)$ & $0.31(0.26-0.40)$ & 0.17 \\
\hline Slope half-life (hours), median (IQR) & $2.36(1.85-2.88)$ & $2.23(1.74-2.63)$ & 0.20 \\
\hline PCT (hours), n & 55 & 54 & \\
\hline PCT, median (IQR) & $30.0(24.0-36.0)$ & $30.0(24.0-36.0)$ & 0.22 \\
\hline PC50, median IQR) & $8.93(4.37-11.84)$ & $7.95(4.65-10.46)$ & 0.77 \\
\hline PC90, median (IQR) & $14.34(10.58-17.78)$ & $14.02(10.65-17.10)$ & 0.51 \\
\hline PC95, median (IQR) & $16.65(13.11-20.69)$ & $16.0(12.78-19.69)$ & 0.44 \\
\hline PC99, median (IQR) & $22.19(18.75-25.84)$ & $21.32(18.46-25.07)$ & 0.34 \\
\hline \multicolumn{4}{|l|}{ Secondary end points } \\
\hline Treatment outcomes, $\mathbf{n}$ & 63 & 57 & \\
\hline LPF (PCR corrected), $n$ & 01 & 00 & I \\
\hline \multicolumn{4}{|l|}{ PCR-corrected cure rate (K-M) } \\
\hline At day $28, \mathrm{n}(\%)(95 \% \mathrm{Cl})$ & $63(100)$ & $57(100)$ & 1 \\
\hline At day $42, \mathrm{n}(\%)(95 \% \mathrm{Cl})$ & 62 (98.07) (88.07-99.67) & $57(100)$ & 1 \\
\hline FCT (hours), (n); median (IQR) & $(63) ; 6(6-6)$ & $(57) ; 6(6-6)$ & 0.49 \\
\hline
\end{tabular}

Notes: ${ }^{\mathrm{P} C} 50,50 \%$ parasite clearance rate; PC $90,90 \%$ parasite clearance rate; PC $95,95 \%$ parasite clearance rate; PC $99,99 \%$ parasite clearance rate. ${ }^{\mathrm{A}} \mathrm{As}$ estimated by the PCE (WWARN).

Abbreviations: AL, artemether + lumefantrine; AS + AQ, artesunate + amodiaquine; FCT, fever clearance time; K-M, Kaplan-Meier; LPF, late parasitological failure; PCE, parasite clearance estimator; PCR, polymerase chain reaction; PCT, parasite clearance time; WWARN, Worldwide Antimalarial Resistance Network. 
Monitoring the efficacy of malaria treatment and studies related to K13 propeller mutations have become essential to detect resistance to ACTs as early as possible.

A significant decrease in the rate of parasite clearance and delayed PCT following treatment with artemisinin and increased failure rates following ACT treatments provided definitive evidence of resistance in Southeast Asia. ${ }^{8,9,24}$

The median PCT in this study was 30 hours (IQR, 24-36 hours), for each ACT, and the median parasite clearance rate had a slope half-life of 2.36 hours (IQR, 1.85-2.88 hours) and 2.23 hours (IQR, 1.74-2.63 hours) for AS + AQ and $\mathrm{AL}$, respectively.

A Phase III trial related to PCT (sampling every 6 hours) from patients treated with AL conducted in the south of Côte d'Ivoire in 2012 and 2014 demonstrated a rapid PCT of 24 hours. ${ }^{20,25}$

It seemed that the current PCT found in the center of the country is higher than in the south. This could be due to patients' immunity related to transmission which is more intense in the south, drug pressure, the type of AL used, human genetics factors, or both.

Most of clinical trials conducted in Côte d'Ivoire since the introduction of $\mathrm{AS}+\mathrm{AQ}$ and $\mathrm{AL}$ as the first-line treatment from 2005 to 2015 measured parasitaemia either daily or only on days 0,2 , and 3 as per WHO guidelines. Results from these studies demonstrated an estimated PCT of 48 hours for both AS + AQ and AL. ${ }^{18,19,26}$ However, these estimations of PCT have some limitations. Determination of PCT based on daily sampling lacks precision since the exact time of parasite count is not recorded. In addition, this time could vary by several hours depending on the timing of the visit to the clinic at inclusion and during the follow-up days. Accurate estimations of parasite clearance rates with WWARN's PCE are suitable but need more frequent samplings which are difficult to implement during routine monitoring of the efficacy of ACTs.

The proportion of patients with persistent parasitaemia on day 3 after ACTs provides a useful indicator to be considered as a simple and readily measure in the setting of drug efficacy surveillance studies. ${ }^{27}$

The delayed parasite clearance at 72 hours is an in vivo predictor of subsequent treatment failure with $\mathrm{ACTs}{ }^{26}$ and an indicator of choice for the routine monitoring of suspected artemisinin resistance in $P$. falciparum.

In the current study, none of the patients presented parasites on day 3 . In a study conducted in 2009 in Côte d'Ivoire, delay in parasite clearance occurred in one patient treated with AL, suggesting probably a decreased sensitivity. ${ }^{26}$
Data related to PCT or proportion of patients with parasitaemia at day 3 from this current study and the previous one showed that malaria patients in Côte d'Ivoire cleared parasites rapidly after ACTs. These results are consistent with those reported from several sub-Saharan African countries. ${ }^{28-30}$ An individual patient data analysis of parasite clearance conducted on a large sample $(n=15,000)$ of uncomplicated malaria treatments showed that rapid $P$. falciparum clearance continues to be achieved in sub-Saharan African patients treated with ACT. ${ }^{31}$

The artemisinin component is mostly responsible for the rapid parasite clearance. Studies conducted in Western Cambodgia on artemisinin alone have reported 84 hours for PCT which was related to artemisinin resistance. ${ }^{12,32,33}$ The PCT with artemisinin observed in African patients is therefore much lower than that found in Western Cambodia. ${ }^{34}$

In a study conducted in Mali where parasitaemia was recorded every 8 hours in patients treated for uncomplicated $P$. falciparum with AS for 7 days and where there was no evidence of delayed parasitemia, the observed median PCT was 32 hours. ${ }^{35}$

Several factors could explain this difference. Indeed, artemisinin has been widely used in Africa since 2000, compared to more than 30 years in Cambodia, and is mainly available as co-formulated $\mathrm{ACT} .^{8,36}$ The other factors are related to host immunity and transmission reservoir of asymptomatic individual. These factors serve as obstacles to the selection of artemisinin resistance. ${ }^{37}$

Factors such as drug concentration, pretreatment parasite density, host malaria-specific immunity, independently of intrinsic drug-related effects, may alter or increase effects of drug on parsites. ${ }^{30-32}$

Malaria-specific immunity factor plays an important role in parasite clearance. A study conducted in the west of Côte d'Ivoire showed that glutamate rich protein and circumsporozoite protein IgG antibody responses in Man region in the west contribute to AS + AQ and AL treatment success and suggest that antimalarial immunity plays an important role in early parasitological responses. ${ }^{38-40}$

Efficacy data from the current study showed a high cure rate for both regimens after a standard day 42 follow-up. PCRcorrected ACPR at day 42 in the per-protocol population was $100 \%$ and $98.07 \%$ for AS + AQ and AL, respectively. Both treatments were well above the WHO recommended $90 \%$ threshold for treatments in use. ${ }^{6}$ Crude clinical treatment failure occurred in ten and two participants in the AL and AS + AQ groups, respectively. Our findings are in line with results from previous studies that showed that ACTs had a high cure rate and short PCT in malaria-endemic areas in Côte d'Ivoire. ${ }^{18-20}$ 
The study has some limitations. Pharmacology of drug action, parasite biology, and human immunity were not performed.

\section{Conclusion}

Patients treated with AS + AQ and AL cleared malaria parasites quickly in Bouake region. Data from this study support evidence that ACTs are still efficacious in this part of Côte d'Ivoire, but continued efficacy monitoring of ACTs is still needed.

\section{Acknowledgments}

We thank all the study participants and local medical staff of Dar Es Salam. This study was a part of PALEVALUT Project funded by the Global Fund to fight HIV, tuberculosis, and malaria via the Initiative 5\% program initiated by the French Ministry of Foreign Affairs and France Expertise Internationale (grant \#12INI109). The sponsor was not involved in the design of the study or collection, analysis, and interpretation of data or in writing the manuscript.

\section{Author contributions}

All the authors participated in design, implementation, analysis, or interpretation of the study. OAT was involved in all phases of the study and had full access to all the data in the study. SBA, AAK, FR, CR, and SB participated in the design and supervised the study. TNL, AAK, BK, EAG, and $\mathrm{OO}$ were responsible for conducting field studies and coordinating study procedures. BAA and BC performed PCR genotyping, and EAG performed the statistical analysis. The manuscript was drafted by OAT; substantial input came from all investigators. All authors contributed toward data analysis, drafting and critically revising the paper, gave final approval of the version to be published, and agree to be accountable for all aspects of the work.

\section{Disclosure}

The authors report no conflicts of interest in this work.

\section{References}

1. WHO. World Health Organization: World Malaria Report 2017. Geneva: World Health Organization; 2017.

2. Wongsrichanalai C, Sibley CH. Fighting drug-resistant Plasmodium falciparum: the challenge of artemisinin resistance. Clin Microbiol Infect. 2013;19(10):908-916.

3. Ashley EA, Dhorda M, Fairhurst RM, et al. Spread of artemisinin resistance in Plasmodium falciparum malaria. $N$ Engl $J$ Med. 2014;371(5):411-423.

4. Taylor SM, Parobek CM, Deconti DK, et al. Absence of putative artemisinin resistance mutations among Plasmodium falciparum in Sub-Saharan Africa: a molecular epidemiologic study. $J$ Infect Dis. 2015;211(5):680-688.
5. Woodrow CJ, White NJ. The clinical impact of artemisinin resistance in Southeast Asia and the potential for future spread. FEMS Microbiol Rev. 2017;41(1):34-48.

6. World Health Organization. Methods for Surveillance of Antimalarial Drug Efficacy. Geneva: World Health Organization; 2009.

7. Ariey F, Witkowski B, Amaratunga C, et al. A molecular marker of artemisinin-resistant Plasmodium falciparum malaria. Nature. 2014;505(7481):50-55.

8. Dondorp AM, Nosten F, Yi P, et al. Artemisinin resistance in Plasmodium falciparum malaria. N Engl J Med. 2009;361(5):455-467.

9. Noedl H, Se Y, Sriwichai S, et al. Artemisinin resistance in Cambodia: a clinical trial designed to address an emerging problem in Southeast Asia. Clin Infect Dis. 2010;51(11):e82-e89.

10. Phyo AP, Nkhoma S, Stepniewska K, et al. Emergence of artemisininresistant malaria on the western border of Thailand: a longitudinal study. Lancet. 2012;379(9830):1960-1966.

11. Kamau E, Campino S, Amenga-Etego L, et al. K13-propeller polymorphisms in Plasmodium falciparum parasites from sub-Saharan Africa. J Infect Dis. 2015;211(8):1352-1355.

12. Boussaroque A, Fall B, Madamet M, et al. Emergence of Mutations in the K13 Propeller Gene of Plasmodium falciparum Isolates from Dakar, Senegal, in 2013-2014. Antimicrob Agents Chemother. 2016;60(1):624-627.

13. Ménard D, Khim N, Beghain J, et al. A Worldwide Map of Plasmodium falciparum K13-Propeller Polymorphisms. $N$ Engl J Med. 2016;374(25):2453-2464.

14. Ogouyèmi-Hounto $\mathrm{A}$, Damien $\mathrm{G}$, Deme $\mathrm{AB}$, et al. Lack of artemisinin resistance in Plasmodium falciparum in northwest Benin after 10 years of use of artemisinin-based combination therapy. Parasite. 2016;23:28.

15. Flegg JA, Guerin PJ, White NJ, Stepniewska K. Standardizing the measurement of parasite clearance in falciparum malaria: the parasite clearance estimator. Malar J. 2011;10:339.

16. Karunajeewa HA. Parasite clearance after malaria therapy: staying a step ahead of drug resistance. BMC Med. 2015;13:251.

17. White NJ. Malaria parasite clearance. Malar J. 2017;16(1):88.

18. Toure OA, Assi SB, N'Guessan TL, et al. Open-label, randomized, noninferiority clinical trial of artesunate-amodiaquine versus artemether-lumefantrine fixed-dose combinations in children and adults with uncomplicated falciparum malaria in Côte d'Ivoire. Malar J. 2014;13:439.

19. Yavo W, Konaté A, Kassi FK, et al. Efficacy and Safety of ArtesunateAmodiaquine versus Artemether-Lumefantrine in the Treatment of Uncomplicated Plasmodium falciparum Malaria in Sentinel Sites across Côte d'Ivoire. Malar Res Treat. 2015;2015(2):1-8.

20. Toure OA, Valecha N, Tshefu AK, et al. A Phase 3, Double-Blind, Randomized Study of Arterolane Maleate-Piperaquine Phosphate vs Artemether-Lumefantrine for Falciparum Malaria in Adolescent and Adult Patients in Asia and Africa. Clin Infect Dis. 2016;62(8):964-971.

21. World Wide Antimalarial Resistance Network. Parasite Clearance Estimator (PCE) WorldWide Antimalarial Resistance Network. Oxford, United Kingdom; 2015. Available from: http://www.wwarn.org/toolsresources/toolkit/analyse/parasite-clearance-estimator-pce. Accessed December 6, 2017.

22. Medicines for Malaria Venture, WHO. Methods and Techniques for Clinical Trials on Antimalarial Drug Efficacy: Genotyping to Identify Parasite Populations; 2008. Available from: http://www.who.int/malaria/ publications/atoz/9789241596305/en/. Accessed December 6, 2017.

23. Felger I, Beck HP. Genotyping of Plasmodium falciparum. PCR-RFLP analysis. Methods Mol Med. 2002;72:117-129.

24. Rogers WO, Sem R, Tero T, et al. Failure of artesunate-mefloquine combination therapy for uncomplicated Plasmodium falciparum malaria in southern Cambodia. Malar J. 2009;8:10.

25. Toure OA, Mwapasa V, Sagara I, et al. Assessment of Efficacy and Safety of Arterolane Maleate-Piperaquine Phosphate Dispersible Tablets in Comparison With Artemether-Lumefantrine Dispersible Tablets in Pediatric Patients With Acute Uncomplicated Plasmodium falciparum Malaria: A Phase 3, Randomized, Multicenter Trial in India and Africa. Clin Infect Dis. 2017;65(10):1711-1720. 
26. Toure Offianan A, Assi SB, Aristide MAC, et al. Assessment of the efficacy of first-line antimalarial drugs after 5 years of deployment by the National Malaria Control Programme in Côte d'Ivoire. Open Access J Clin Trials. 2011;3:67-76.

27. Stepniewska K, Ashley E, Lee SJ, et al. In vivo parasitological measures of artemisinin susceptibility. J Infect Dis. 2010;201(4):570-579.

28. WWARN Artemisinin based Combination Therapy (ACT) Africa Baseline Study Group, Dahal P, D’Alessandro U, et al. Clinical determinants of early parasitological response to ACTs in African patients with uncomplicated falciparum malaria: a literature review and meta-analysis of individual patient data. BMC Med. 2015; $13: 212$.

29. Muhindo MK, Kakuru A, Jagannathan P, et al. Early parasite clearance following artemisinin-based combination therapy among Ugandan children with uncomplicated Plasmodium falciparum malaria. Malar J. 2014;13:32.

30. Das D, Price RN, Bethell D, Guerin PJ, Stepniewska K. Early parasitological response following artemisinin-containing regimens: a critical review of the literature. Malar J. 2013;12:125.

31. Zwang J, Dorsey G, Mårtensson A, et al. Plasmodium falciparum clearance in clinical studies of artesunate-amodiaquine and comparator treatments in sub-Saharan Africa, 1999-2009. Malar J. 2014;13: 114-119.

32. Amaratunga C, Sreng S, Suon S, et al. Artemisinin-resistant Plasmodium falciparum in Pursat province, western Cambodia: a parasite clearance rate study. Lancet Infect Dis. 2012;12(11):851-858.
33. Vreden SG, Bansie RD, Jitan JK, Adhin MR. Assessing parasite clearance during uncomplicated Plasmodium falciparum infection treated with artesunate monotherapy in Suriname. Infect Drug Resist. 2016;9:261-267.

34. WWARN Parasite Clearance Study Group, Abdulla S, Ashley EA, et al. Baseline data of parasite clearance in patients with falciparum malaria treated with an artemisinin derivative: an individual patient data metaanalysis. Malar J. 2015;14:359.

35. Maiga AW, Fofana B, Sagara I, et al. No evidence of delayed parasite clearance after oral artesunate treatment of uncomplicated falciparum malaria in Mali. Am J Trop Med Hyg. 2012;87(1):23-28.

36. Maude RJ, Pontavornpinyo W, Saralamba S, et al. The last man standing is the most resistant: eliminating artemisinin-resistant malaria in Cambodia. Malar J. 2009;8:31.

37. Nyunt MM, Plowe CV. Pharmacologic advances in the global control and treatment of malaria: combination therapy and resistance. Clin Pharmacol Ther. 2007;82(5):601-605.

38. Ndour PA, Lopera-Mesa TM, Diakité SA, et al. Plasmodium falciparum clearance is rapid and pitting independent in immune Malian children treated with artesunate for malaria. J Infect Dis. 2015;211(2):290-297.

39. Yao SS, Offianan AT, Tiacoh NL. Associations between IgG antibody responses to multiple Plasmodium falciparum antigens and treatment outcomes with ACTs in Man, Cote d'Ivoire. Afr J Parasitol Res. 2017;4(7):234-243.

40. Lopera-Mesa TM, Doumbia S, Chiang S, et al. Plasmodium falciparum clearance rates in response to artesunate in Malian children with malaria: effect of acquired immunity. J Infect Dis. 2013;207(11):1655-1663.
Infection and Drug Resistance

\section{Publish your work in this journal}

Infection and Drug Resistance is an international, peer-reviewed openaccess journal that focuses on the optimal treatment of infection (bacterial, fungal and viral) and the development and institution of preventive strategies to minimize the development and spread of resistance. The journal is specifically concerned with the epidemiology of antibiotic

\section{Dovepress}

resistance and the mechanisms of resistance development and diffusion in both hospitals and the community. The manuscript management system is completely online and includes a very quick and fair peerreview system, which is all easy to use. Visit http://www.dovepress.com/ testimonials.php to read real quotes from published authors. 